\title{
Alter
}

Revue de phénoménologie

$19 \mid 2011$

Le langage

\section{Mettre en mots les choses elles-mêmes : le difficile passage de l'expérience à l'expression}

Noémie Parant

\section{(2) OpenEdition}

\section{Journals}

Édition électronique

URL : http://journals.openedition.org/alter/1411

DOI : 10.4000/alter.1411

ISSN : 2558-7927

Éditeur :

Association ALTER, Archives Husserl (CNRS-UMR 8547)

\section{Édition imprimée}

Date de publication : 1 octobre 2011

Pagination : 157-179

ISBN : 978-2-9522374-7-5

ISSN : 1249-8947

\section{Référence électronique}

Noémie Parant, « Mettre en mots les choses elles-mêmes : le difficile passage de l'expérience à

l'expression », Alter [En ligne], 19 | 2011, mis en ligne le 01 janvier 2020, consulté le 23 janvier 2020

URL : http://journals.openedition.org/alter/1411; DOI : 10.4000/alter.1411 


\section{METTRE EN MOTS LES CHOSES ELLES-MÊMES : LE DIFFICILE PASSAGE DE L'EXPÉRIENCE À L'EXPRESSION}

Noémie Parant

«Le Compte-tenu des Mots ferait assez bien le pendant du Parti-pris des Choses ${ }^{1}$

\section{Préambule}

L'idée, en ces lignes, est d'explorer la liaison qui unit l'expression phénoménologique à l'expérience - et, ainsi, les mots aux choses. Sachant que c'est au fil conducteur de la perspective husserlienne qu'il s'agit d'opérer; sachant aussi qu'il est question de lire en le couple expression-expérience un rapport particulier - puisque l'on ne veut voir en le langage du phénoménologue rien autre $\mathrm{qu}^{\prime} u n e$ expression de l'expérience subjective.

Certes, on ne niera pas que cette façon d'appréhender l'écriture phénoménologique peut sembler $s^{\prime}$ inscrire à rebours du projet principiel de la discipline en présence : parce qu'il n'est pas question, dans l'économie de la pensée husserlienne, de traiter de la parole employée en phénoménologie ni de la dicibilité des choses ellesmêmes (Husserl, en effet, n'examine pas sa propre " pratique [...] de l'écriture $[\ldots]$. La non-thématisation du langage est constitutive de la phénoménologie, qui prend pour thème le sens des phénomènes et non leur articulation langagiere $»^{2}$ ) ni, a fortiori, du langage phénoménologique comme possibilité de dire ces choses. Pourtant, la

\footnotetext{
1. F. Ponge, CEuvres complètes, II, Paris, Gallimard, coll. "Bibliothèque de la Pléiade », 2002, édition publiée sous la direction de Bernard Beugnot, avec, pour ce volume, la collaboration de G. Farasse, J.-M. Gleize, J. Martel, p. 1188.

2. N. Depraz, Écrire en phénoménologue, "Une autre époque de l'écriture », La Versanne, Encre Marine, Fougères, 1999, p. 22.
} 
volonté de traiter de l'expression de l'expérience en phénoménologie, volonté qui peut paraître non-husserlienne par nature, n'est pas arbitraire. De fait, il suffit de se pencher sur certains des textes de Husserl pour se rendre compte qu'il l'évoque lui-même : ainsi fait-il état de la possibilité de mettre des mots sur les choses elles-mêmes. À titre d'illustration, on relèvera simplement cette phrase : «Naturellement cela n'exclurait pas qu'il y ait plusieurs manières d'exprimer par exemple des vécus affectifs $»^{3}$, en tant qu'elle atteste qu'il est bien question, avec Husserl même, de dire le "vivre".

Seulement, en quoi consiste précisément cette opération? Que signifie, rigoureusement, dire le "vivre" ? Encore faut-il donc ressaisir avec acuité cette formulation des choses, ici pressentie, au sein des textes phénoménologiques.

\section{L'expression de l'expérience}

Pour ce faire, il n'est que d'être attentif à la textualité en jeu : car on découvre d'emblée en elle en quel sens elle est une mise en mots du champ expérientiel - ou " une "langue phénoménale" de l'expérience immédiate $»^{4}$. Prenons pour exemple ces quelques lignes de Husserl :

Je vois continuellement cette table; j'en fais le tour et change comme toujours ma position dans l'espace; j'ai sans cesse conscience de l'existence corporelle d'une seule et même table, de la même table qui en soi demeure inchangée. Or la perception de la table ne cesse de varier; c'est une série continue de perceptions changeantes. Je ferme les yeux. Par mes autres sens je n'ai pas de rapport à la table. Je n'ai plus d'elle aucune perception. J'ouvre les yeux et la perception reparaît de nouveau ${ }^{5}$.

Ces lignes, en effet, prennent la forme d'un langage du phénoménal en cela que le vécu perceptif de table qui y est mis en mots l'est de telle sorte qu'il vient émerger au sein du discours : il se loge dans les voies du langage et y jaillit avec force. Il semble même qu'il soit question ici d'effectuer l'expérience, et ce, au travers de l'expression : parce que les mots y sont manifestement habités par l'épaisseur du "vivre", au point de délivrer la présence vivante de cette densité. De fait, il y a là quelque chose qui se manifeste dans l'écriture, et qui est précisément le manifeste par excellence ; de fait encore, les apparitions se phénoménalisent ici à même la textualité - comme si les vécus s'imposaient à l'espace langagier, et venaient hanter le langage

\footnotetext{
3. E. Husserl, Idées directrices pour une phénoménologie et une philosophie phénoménologique pures, I, «Introduction générale à la phénoménologie pure », Paris, Gallimard, coll. « Tel », 2003, trad. P. Ricœur, cité d'après la pagination allemande, p. 263.

4. A. Soulez, Comment écrivent les philosophes, «(De Kant à Wittgenstein) ou le style Wittgenstein », Éditions Kimé, Paris, 2003, p. 260.

${ }^{5}$. E. Husserl, Idées directrices pour une phénoménologie et une philosophie phénoménologique pures, I, op. cit., p. 73-74.
} 
en formulant le vœu de « $\mathrm{s}^{\prime}$ installe[r] alors au cœur même du dire » $^{6}$. On comprend donc, à partir de cet exemple, en quoi consiste précisément l'expression de l'expérience : il s'agit d'une expression dont la marque est de se trouver ressaisie par l'expérience qu'elle exprime de $s^{\prime}$ en trouver habitée jusqu'au sein de ses creux et de ses reliefs : jusqu'au cœur des silences et des mots qui la composent. Par conséquent, le phénoménologue traduit en mots les choses elles-mêmes dès lors qu'il s'attache, dans sa propre écriture, à faire émerger ces choses et à en offrir une transcription ; dès lors que son propos, en d'autres termes, a pour marque frappante celle de contenir la sphère du vécu et de se doter de son éclatante trame. En somme, parler phénoménologiquement de l'apparaître c'est intégrer l'expérience dans le domaine du dicible et l'inscrire dans l'espace langagier : c'est mettre en place un langage dont la spécificité est d'avoir exhaussé jusqu'à soi les choses elles-mêmes, dans un acheminement lumineux vers la dicibilité - l'essentiel en la matière étant ce transport des choses au cœur des mots et, partant, la couleur particulière que prennent ces derniers dès l'instant où ils portent en eux l'expérience. Ainsi faut-il en conclure que la tentative de dire l'expérience de la conscience, dans un cadre phénoménologique tout du moins, aboutit à la présence insistante et presque envahissante de cette expérience dans le "dire" : on le voit, l'apparaître se formule de se concentrer dans le discours qui le formule - de s'insérer, donc, dans la discursivité et de s'introduire dans toutes ses ramifications.

De cette façon, l'écriture phénoménologique est écriture de l'expérience au sens où sa syntaxe, sa forme et son vocabulaire permettent de rencontrer l'expérience qu'elle s'attache à restituer: elle l'est au sens où elle a pour qualité particulière de se tenir au plus près de la sphère phénoménale - chacun des mots employé ou chacune des avancées langagières effectuée y étant toujours l'occasion de se poster à proximité de l'apparaître. L'expression de l'expérience est telle, en bref, de se prêter à l'incarnation de cette experience même en en endossant la chair. Mais que déduire de cette réflexion? De ce constat, plutôt, selon lequel la parole phénoménologique exprime l'expérience dès lors que l'expérience vient se lover en elle et y déposer son étoffe? Le fait qu'il se produit alors un mélange des matières langagière et expérientielle : en effet, à partir du moment où «les mots que nous employons pour exprimer tel état de choses sont faits de la même pâte que ce que nous voulons exprimer par leur moyen [en l'occurrence, les phénomènes] $\gg^{7}$, il ne peut qu'y avoir amalgame des tissus discursif et phénoménal, voire identité des intonations et des accents qu'ils revêtent l'un et l'autre. Autrement dit, l'apparaître

${ }^{6}$. J. Benoist, L'idée de phénoménologie, Paris, Beauchesne, coll. « Le grenier à sel », 2001, p. 66.

7. A. Soulez, Comment écrivent les philosophes, op. cit., p. 209. 
s'exprime d'habiter l'expression en lui conférant ses propres traits, mais de sorte qu'il ne peut alors se produire qu'un recouvrement - le "dire" et le "vivre" ne faisant plus qu'un ; les mots cessant d'être d'un côté et les choses de l'autre, pour substituer à ce départage de simples "mots-choses". Cela signifie que des mots aux choses il n'y a alors, en phénoménologie, pas grande différence: parce que s'enquérir des mots ici, c'est déjà presque s'enquérir des choses ; c'est s'efforcer de rompre la distance entre le "dire" et le "vivre" en procédant de telle manière que « le langage [soit] à peine séparé du phénomène » et que « le mot [soit] tout contre la chose, comme s'ils étaient bord à bord $»^{8}$. Partant, toute ligne de démarcation entre l'expérience et l'expression s'étant éteinte, il n'est question à ce niveau que de continuité et de correspondance strictes : il n'est question, en somme, que de concordance et de superposition entre "ce qui se vit" et "ce qui se dit". En termes temporels, termes qui sont ici les plus à même d'expliciter ce qu'il se produit, on peut dire qu'une rigoureuse simultanéité s'instaure entre les mots et les choses: on peut soutenir même que la phénoménologie exprime l'expérience de découvrir l'isochronisme entre l'expérience et l'expression et d'instaurer « une coïncidence de l'expérience vécue et de l'expression verbale qui lui correspond $»^{9}$. Coïncidence que Husserl a d'ailleurs lui-même thématisée, d'une thématisation qui légitime en définitive notre propos: "Les mots employés peuvent être issus de la langue commune, $[\ldots]$ : tant qu'ils "coïncident" (sich decken) avec le donné intuitif sous la forme d'une expression actualisée, ils prennent un sens déterminé, [...] ; à partir de ce moment ils peuvent être fixés scientifiquement $»^{10}$.

\section{L'expérience (in-)exprimable?}

Or, on ne peut pas nier que cette analyse fait fond sur une présupposition: elle contient la théorie sous-jacente que l'expérience bénéficie d'une certaine capacité à s'inscrire dans le langage. Tout se déroule, effectivement, comme si les apparitions ne manifestaient aucune réticence à l'égard du discours : à l'inverse, elles y semblent disposées, d'un penchant irréductible. Car comment comprendre autrement le fait qu'elles puissent être traduites langagièrement, d'une traduction qui va jusqu'à la coïncidence? Cela suppose nécessairement qu'elles détiennent en leur nature, plus qu'une tendance, une véritable vocation au langage : il ne peut y avoir de parfaite continuité du "voir" au "dire", sans l'idée que le "voir" lui-même soit destiné au "dire". Radicalement, cela signifie que l'apparaître s'impose comme un espace

\footnotetext{
8. Ibid., p. 102.

9. N. Depraz, Écrire en phénoménologue, op. cit., p. 107.

10. E. Husserl, Idées directrices pour une phénoménologie et une philosophie phénoménologique pures, I, op. cit., p. 124.
} 
toujours prêt à être mis en mots - et, en un sens : toujours déjà dit. Qui plus est, s'il bascule ainsi sans résistance dans l'ordre du dicible, c'est forcément parce qu'il est lui-même un lieu de langage possible. On est porté à croire, en ce sens, que le vécu n'est pas seulement un espace auquel on peut appliquer la discursivité et qui fait état du desir d'être dit, mais qu'il est aussi au principe d'un discours particulier : qu'il parle lui-même donc, d'une parole qui ne fait jamais que témoigner de l'existence d'un "langage de l'expérience » ${ }^{11}$.

Pour autant, ce qui semble ici aller de soi, ne va pas sans poser de difficultés : c'est que l'idée même d'une discursivité expérientielle est problématique. Elle ne laisse pas, en tout cas, de susciter la perplexité : parce qu'à première vue, du moins, le fait de vivre ne paraît pas toujours corrélé à celui de parler. En effet, éprouver, est-ce forcément s'installer dans la sphère langagière ? Est-ce constamment se formuler à soi-même et, a fortiori, aux autres ce que l'on vit? L'émergence même de ces questions atteste que le problème du rapport entre le langage et les choses elles-mêmes n'a pas encore été complètement exploré : tout un pan en demeure, à ce stade, obscur et confus. Aussi, il faut désormais s'engager au cœur de ce versant énigmatique, et prendre acte avec sérieux des interrogations ci-dessus mises en lumière : autrement dit, tout repose à présent dans cette nuance selon laquelle l'expérience n'est pas nécessairement un champ d'expression - elle peut l'être, oui, mais à ceci près que, de la possibilite à la nécessité, il y a un pas immense. C'est cette distance, en laquelle réside la subtilité du propos, qu'il convient d'assumer jusqu'au bout: toute la réflexion à venir doit donc prendre son départ dans le constat que l'expérience peut basculer dans l'expression, d'une possibilité qui n'est pas une nécessité et qui, comme telle, laisse ouverte d'autres possibilités - en l'occurrence, celle selon laquelle l'expérience puisse se refuser, justement, à verser dans l'expression.

\section{II}

\section{Préambule}

Ainsi découvre-t-on une incertitude concernant l'accord entre les mots et les choses. C'est qu'il n'est pas certain, en effet, que l'expérience et l'expression se rencontrent, soient en mesure plutôt, autant l'une que l'autre, de se rencontrer. Car, en somme, les phénomènes sont-il dicibles? Appartiennent-ils au champ langagier? La possibilité demeure, on le voit, que les vécus soient en fait promis au silence - d'une promesse qui rend difficile à soutenir l'idée d'un

\footnotetext{
11. «Le langage de la création esthétique dans la phénoménologie », M. Sancipriano, Analecta Husserliana, vol. XXXIII, 1991, p. 283.
} 
langage des choses elles-mêmes, mis en place par le phénoménologue. Assumons pourtant cette possibilite : c'est la meilleure façon de trouver une solution en la matiere. Prenons donc le parti de penser avec rigueur cette indicibilité de l'expérience qui émerge ici : car, après tout, si jamais le discours est écarté à l'endroit des phénomènes, ne serait-ce pas simplement un certain type de discours? De sorte que tout discours ne serait en fait pas exclu et que l'idée d'une expression phénoménologique des phénomènes resterait envisageable ? Mais sous quelles conditions le resterait-elle c'est-à-dire à condition que le phénoménologue emploie ou retienne comme sien quel type de langage?

\section{Hors du langage : les phénomènes}

Dans cette optique, mettons l'accent pour commencer sur le fait que les vécus se refusent effectivement à l'expressivité, de sorte que leur retrait du langage n'est pas seulement possible mais bel et bien réel: de sorte, surtout, qu'ils témoignent d'une appartenance à la sphère de l'indicible. D'ailleurs, on notera que l'apparaître s'engage même à maintenir son caractère inexpressif : il persiste, actuellement pourrait-on dire, à être silencieux. C'est toute la signification du "encore muette" dans la fameuse affirmation de Husserl selon laquelle "c'est l'expérience pure et, pour ainsi dire, muette encore, qu'il s'agit d'amener à l'expression pure de son propre sens ${ }^{12}$. Le "encore" ici employé se comprend en effet comme le corrélat d'un certain "pas encore" : c'est que les phénomènes appartiennent encore à l'ineffable de telle sorte qu'ils n'appartiennent pas encore au dicible - ils relèvent toujours du non-dit du fait, rigoureusement, qu'ils n'ont actuellement toujours pas accédé au dire, ni même à son seuil.

Or, ce maintien dans le non-encore langage a une signification spécifique: les vécus ne sont toujours pas parvenus à atteindre la parole au sens où ils persistent à s'inscrire dans un silence qui prend la forme singulière d'un "en deçà du langage »13. Par conséquent, ils résident dans un non-dit particulier, dont la particularité est de s'étendre en amont du langage : là est le lieu où ils prennent corps et $s^{\prime}$ installent - s'imposant comme ce qui précède et se tient avant la parole. Les choses elles-mêmes, de ce fait, sont muettes d'être le prélude à tout discours : car leur manifestation revendique le titre de préalable au langage. Ainsi Husserl avance-t-il que notre vie est prélangagière $^{14}$; ainsi aussi soutient-il l'existence de « L'expérience anté-

\footnotetext{
12. E. Husserl, Méditations cartésiennes, Paris, Vrin, 1996, trad. G. Peiffer et E. Lévinas, cité d'après la pagination de la traduction, p. 73-74.

13. N. Depraz, Écrire en phénoménologue, op. cit., p. 19.

14. Cf. E. Husserl, De la synthèse passive, "Logique transcendantale et constitutions originaires ", Grenoble, Jérôme Millon, coll. "Krisis », 1998, trad. B. Bégout et J. Kessler avec la collaboration de N. Depraz et M. Richir, Introduction : «Considérations préparatoires au Cours sur la logique
} 
prédicative » qui, par nature, se situe avant «La couche tout entière de l'expression qui est [...] inséparablement liée aux opérations prédicatives ${ }^{15}$. En l'occurrence, cette reconduction des vécus à l'en deçà du langage implique sensiblement que ces mêmes vécus admettent quelque rapport avec le niveau de l'implicite : parce que l'appartenance à la sphère logée sous la discursivité recouvre celle de l'appartenance au sous-entendu. De cette façon, on peut dire que l'expérience a pour marque de partager son espace avec celui du tacite : manifestement, elle habite les dessous du langage, c'est-à-dire tous ces îlots in-explicites qui hantent la parole dévoilee ; c'est-à-dire aussi tous ces creux qui traversent le discours et jaillissent entre les mots. Éprouver, en définitive, c'est donner le privilège à ce qui rejette la possibilité de l'explicite : c'est se situer dans les cavités qui trouent le discours, et scindent l'expression déployée. Le surgissement du "vivre" se fait donc sous les mots, ou derrière ceux-ci - et sans doute aussi dans leurs alentours, lesquels alentours cernent ces mêmes mots à la lisière des frontières qui les délimitent. En d'autres termes, les vécus logent dans cet espace en deçà du langage, qui est le lieu même de l'approximatif et du flou par excellence - où le dit n'est jamais totalement dit. C'est en tout cas ce que laisse à penser Husserl lorsqu'il décrit la conscience et ses vécus comme des espaces fluants, espaces dont il affirme que la formulation ne peut avoir lieu qu'au moyen d'une parole elle-même fluante : aussi, « on ne peut songer à imposer un concept et une terminologie fixes à chacun de ces concreta fluants ${ }^{16}$. C'est effectivement ce qu'il laisse à penser car, en soulignant que l'expérience subjective appelle une expression mouvante et sans fixité, il s'évertue simplement à signaler que cette même expérience convoque le langage de seulement en convoquer le niveau implicite. Cela suppose, d'ailleurs, que les phénomènes s'instituent par rapport à la discursivité en tant, justement, que domaine nonmanifeste : parce qu'ils sont précisément ce qui n'est pas dit ni révélé dans le dire. Avec eux, on a affaire à cette couche particulière du langage qui se situe en arrière de lui-même, où tout est encore à l'état de non-explicité et d'inavoué : à cette strate derrière les paroles où tout se fait sur le mode de la non-monstration - ce lieu de langage qui, pour le dire radicalement, n'en est pas un à proprement parler. Car il est, par définition, un refus de l'expression - étant entendu que

\footnotetext{
transcendantale », IX. « Retour du logos théorique à la vie de conscience pré-théorique, donatrice de sens".

${ }^{15}$. E. Husserl, Expérience et jugement, «Recherches en vue d'une généalogie de la logique », Paris, Puf, coll. «Épiméthée ", 1991, trad. D. Souche-Dagues, cité d'après la pagination allemande, p. 73 et p. 234.

16. E. Husserl, Idées directrices pour une phénoménologie et une philosophie phénoménologique pures, I, op. cit., p. 140. Cf. à ce sujet N. Depraz, Lire Husserl en phénoménologue, "Idées directrices pour une phénoménologie (I) », Paris, Puf, 2008, p. 133-135.
} 
cette dernière a pour sens étymologique de s'imposer comme un "faire sortir de" ; étant entendu donc qu'exprimer c'est, conformément au latin expressio de exprimere, rendre manifeste et s'établir dans le champ de l'apparition ou du dévoilement. Ce champ qui, à l'évidence, n'est pas celui de l'expérience - cette dernière se logeant, on le voit, dans le non-apparu ou le non-dévoilé du langage c'est-à-dire dans ce qui, comme tel, n'est pas un espace de langage.

Plus loin, cette non-appartenance à la sphère discursive, qui prend la forme ici d'une infiltration dans l'en deçà du discours, peut aussi s'entendre comme insertion dans l'au-delà du langage. L'expérience, en effet, témoigne d'un retrait vis-à-vis de toute formulation langagière en cela qu'elle s'institue dans des espaces qui, d'une façon ou d'une autre, se développent à distance de la parole : aussi, éprouver cela peut signifier s'installer en amont de l'expression mais également en aval de celle-ci. Les vécus, en ce sens, ne sont pas seulement en position de précédence par rapport à la mise en mots : la possibilité est aussi qu'ils s'inscrivent dans ce qui constitue "l'après" des mots, c'est-à-dire dans ce domaine particulier qui surgit postérieurement et ultérieurement au langage. Autrement dit, les phénomènes se manifestent au lieu même où l'expression s'éteint d'avoir accédé à son propre aboutissement : ils jaillissent donc au cœur de cet espace qui dépasse toujours déjà le dire, et qui s'institue lorsque la dicibilité s'achève. Par conséquent, l'apparaître fait part de son propre débordement eu égard à la discursivité : il est ce qui, sans conteste, la déborde en ceci qu'il se présente sous le titre d'au-delà du langage ainsi peut-on dire, et soutenir avec force, que l'expérience " excède toujours son expression langagière $»^{17}$. Cette notion d'excès, d'ailleurs, n'est pas insignifiante : elle s'applique à traduire ce que les choses elles-mêmes ont d'essentiellement irréductible. Car elle souligne le fait que les vécus échappent à la prise que le discours tente d'opérer sur eux : il y a ici l'idée que le "vivre" se refuse à être capturé par le langage. Il s'affirme, justement, de telle sorte qu'il se soustrait à toute saisie qui tenterait de s'opérer sur lui-même : en bref, l'évidence est que le champ de l'"éprouver" témoigne d'une transcendance vis-à-vis de la parole, et de la tentative d'emprise que le langage souhaiterait réaliser par sa propre mise en mots. Or, cette limitation du pouvoir langagier par l'expérience peut se traduire également en termes de décalage: l'expression s'essaie à dire les vécus, mais elle se heurte à l'évidence que ces derniers refusent de se laisser enserrer dans le champ étroit des paroles - révélant alors que, des mots aux choses elles-mêmes, il y a une non-correspondance. C'est que le discours dont l'objectif est de restituer l'apparaître découvre toujours, au cours de sa mise en ouvre effective, 1'impossibilité

17. N. Depraz, Écrire en phénoménologue, op. cit., p. 104. 
d'une juste restitution : le dire rencontre, plutôt que le vécu lui-même, l'illusion qu'il y a à croire $\mathrm{s}^{\prime} \mathrm{y}$ superposer et y concorder rigoureusement. Cette affirmation, en l'occurrence, s'inscrit dans une économie de pensée husserlienne : il n'est que de se référer à la manière dont Husserl envisage le rapport existant entre l'expression et l'exprimé. Selon lui, il n'y a pas d'ajustement point par point entre le langage et ce sur quoi il porte : l'objectité traduite en mots dévoile sa non-conformité à la sphère langagière - et, par là, sa résistance à s'y laisser ramener, sous la forme d'une coïncidence stricte. Aussi: «Toute expression, non seulement énonce quelque chose, mais énonce encore sur quelque chose; elle n'a pas seulement sa signification, mais elle se rapporte aussi à des objets quels qu'ils soient. [...]. Mais jamais l'objet ne coïncide avec la signification $»^{18}$.

En définitive, si l'on prend Husserl comme fil conducteur, il apparaît que l'expérience ne cesse de s'établir dans les marges du langage : soit elle s'ancre en deçà des mots, soit elle s'implante audela de ceux-ci. Dans tous les cas, on aboutit à la même conclusion : vivre, c'est forcément s'abandonner à une dimension dont la spécificité est d'habiter aux franges du dicible. C'est forcément plonger dans les bordures du discours, lesquelles bordures sont à comprendre comme extérieures : car la présence de l'“éprouver" aux frontières du langage ne signifie pas sa quelconque introduction dans le champ langagier ; car le vécu est autour de la parole, attestant son appartenance à ses entours de ne pas lui appartenir, justement. De l'en deçà à l'au-delà se dessine donc une sphere échappant au langage d'une manière ou d'une autre, loin de lui revenir: la sphère phénoménale qui a toujours déjà fui le discours, en se présentant comme intraduisible en langage.

D'ailleurs, ce refus de se laisser traduire en langage dont l'expérience témoigne est à entendre radicalement, d'une radicalité qui $\mathrm{s}^{\prime}$ atteste de deux façons : tentons de l'attester, en prenant légèrement appui sur Heidegger. De la sorte, soulignons d'abord que le silence des choses elles-mêmes ne doit pas être appréhendé comme un mode du langage articulé : il n'est pas ce type d'inexprimable qui s'affirme in-dicible certes, mais qui n'est jamais que la promesse du dicible. En bref, le mutisme des vécus n'endosse pas le sens du silence heideggerien, lequel n'est pas l'antipode de la parole mais plutôt ce qui en constitue le lieu le plus essentiel, et en tout cas un mode possible

\footnotetext{
18. E. Husserl, Recherches logiques, tome 2 : «Recherches pour la phénoménologie et la théorie de la connaissance », première partie (Recherches I et II), Paris, Puf, coll. " Épiméthée », 1972, trad. H. Élie, A. L. Kelkel et R. Schérer, cité d'après la pagination allemande, p. 46. On notera ici l'existence d'un certain paradoxe chez Husserl, puisque l'on a vu par ailleurs qu'il soutient $a$ contrario l'idée d'une coïncidence expérience / expression.
} 
voire fondamental de celle-ci ${ }^{19}$. C'est en ce sens que l'expérience prend une distance radicale par rapport à l'expression: il y a radicalité parce qu'elle refuse d'être une possibilité parmi d'autres du langage articulé. Qui plus est, il y a radicalité en ce sens là aussi que les phénomènes n'instaurent pas seulement un retrait eu égard à la parole articulée mais aussi vis-à-vis du langage tout court - y compris, par conséquent, vis-à-vis des formes de langages non-articulés. En effet, le fait est que la sphère des phénomènes exclut la possibilité de l'articulation, de telle sorte que les mots ne peuvent y surgir : mais le fait est également que cette sphère se refuse même à la possibilité de l'expression - loin de se contenter d'en rejeter seulement la configuration verbale. À proprement parler, on ne peut donc pas soutenir l'idée que l'espace des vécus est un lieu de langage, même si par ce dernier terme on voulait suggérer une dicibilité qui se passe de sons prononcés ou figurés graphiquement. Ainsi, pour reprendre les mots de Heidegger, le "vivre" est silencieux en cela qu'il " n'est pas que et $n^{\prime}$ est surtout pas premièrement - [...] expression orale et écrite de ce qui doit être communiqué $\gg^{20}$, mais aussi en cela qu'il n'est tout simplement pas expression - que celle-ci soit ou non orale et écrite.

Au seuil de cette réflexion, il apparaît désormais clair que l'expérience est ineffable : on a effectivement vu qu'elle est muette au sens où elle habite l'avant tout autant que l'après du langage et qu'elle l'est, en outre, d'un mutisme radical parce qu'ir-reconductible au langage articulé comme au langage tout court. Que peut-on en conclure? Simplement le fait que vivre c'est faire vœu de silence: qu'éprouver c'est éprouver de ne pas parler et de s'inscrire dans des espaces extérieurs au langage. Simplement le fait donc qu'il y a extériorité de l'expérience eu égard à la discursivité. On le voit en effet, on s'est appliqué plutôt à le voir tout au long de l'analyse : les choses elles-mêmes sont telles qu'elles s'établissent en dehors du langage et qu'elles résistent à la possibilité de venir se loger en son dedans; elles sont telles qu'elles s'imposent comme étrangères à la parole, d'une étrangèreté qui ne se laisse pas ramener à l'intimité. $C^{\prime}$ est donc à ce constat qu'il s'agit de s'arrêter : celui selon lequel les phénomènes s'imposent toujours comme "phénomènes hors langage ${ }^{21}$. Or, ce constat même n'est pas sans conséquences sur l'atti-

\footnotetext{
19. «Ce n'est pas sur un autre fondement existential que repose une deuxième possibilité essentielle du parler, le faire-silence. Celui qui fait-silence dans l'être-l'un-avec-l'autre peut "donner" plus véritablement à "comprendre", autrement dit mieux configurer la compréhension que celui qui ne se défait jamais de la parole » (M. Heidegger, Être et temps, Éditions Authentica, 1985, trad. E. Martineau, cité d'après la pagination de la dixième édition, Niemeyer, 1963, p. 164).

${ }^{20}$. M. Heidegger, "L'origine de l'œuvre d'art", in Chemins qui ne mènent nulle part, Paris, Gallimard, 2004, trad. W. Brokmeier, cité d'après la pagination de la traduction, p. 82-83.

${ }^{21 .}$ "Phénoménologie, métaphysique et poïétique», M. Richir, Études phénoménologiques, "Phénoménologie et poétique », no 5-6, 1987, p. 91.
} 
tude à adopter lorsque l'on se trouve face au champ phénoménal : car si le mutisme est ce qui caractérise ce champ alors on est tenu de respecter cette appartenance à l'indicible - si tant est, à tout le moins, que l'on a pour projet de s'en tenir aux choses elles-mêmes. On est tenu, en somme, d'accepter que le langage a aussi des limites, non pas par résignation mais plutôt par célébration des phénomènes - dans le vœu, en tout cas, de leur rendre justice. De la sorte, il faut alors bien garder à l'esprit l'évidence que l'expérience est muette par essence : il faut refuser de satisfaire au désir impatient de se prononcer - en reconnaissant que l'on n'a affaire ici qu'à de l'inarticulé ; en reconnaissant aussi que notre ambition de départ, celle de lier les choses elles-mêmes à la dimension langagière, ne va décidément pas de soi. En bref, telle est la question que l'on doit, selon toute analyse, se poser en présence des vécus - et ce, avant même que la tentation du discours ne commence de s'exercer: "Le vécu, [...] qui échappe à la prise, se laisse-t-il fixer dans le signe? $\gg^{22}$. A titre de réponse, et en accord avec Wittgenstein ${ }^{23}$, c'est une injonction au mutisme que l'on doit alors se formuler à soi-même : parce que le tout, en la matière, est de ne pas astreindre l'indicible au langage; parce que la difficulté, qui s'impose comme un commandement, est de résister au souhait d'assujettir les vécus. C'est dire, donc, que la règle en présence des phénomènes consiste à cultiver l'ineffable et à lui obéir, au lieu de le contraindre à s'exprimer - et à se nier dans sa nature, pourtant éblouissante, d'inexprimable.

\section{La critique du discours de la philosophie traditionnelle}

Cette conclusion, en l'occurrence, est particulièrement problématique si l'on se place du point de vue du phénoménologue : il est en effet évident qu'il ne peut qu'être affecté au plus haut point par cette idée selon laquelle les choses in persona sont silencieuses par définition. Car la question pour lui n'est désormais plus seulement de retourner aux choses elles-mêmes, mais d'y retourner du mouvement même par lequel il se détourne alors du langage. C'est qu'une relation d'opposition s'instaure entre les vécus et la discursivité : on l'a vu, le champ de l'apparaître se refuse au langage et, a fortiori, à sa propre dicibilité - telle est l'évidence que le phenoménologue s'attache justement à ressaisir : ainsi, " La phénoménologie rend un service important en montrant que la chose, la chose proprement dite,

\footnotetext{
22. L. Wittgenstein, Dictées de Wittgenstein à Friedrich Waismann et pour Moritz Schlick, 1, textes inédits (années 1930), Paris, Puf, coll. « Philosophie d'aujourd'hui », 1997, traductions de l'allemand selon des textes transcrits à partir de matériaux dictés par Wittgenstein à $\mathrm{F}$. Waismann et pour M. Schlick établis par G. Baker avec le concours de B. McGuinness, p. 157.

23. L. Wittgenstein, Tractatus logico-philosophicus, Paris, Gallimard, coll. «Bibliothèque de Philosophie », 1993, trad. G.-G. Granger, p. 112 : «Sur ce dont on ne peut parler, il faut garder le silence ».
} 
authentique, in persona, est la chose [...] telle qu'elle s'oppose à [...] l'allusion verbale, quand nous en parlons ${ }^{24}$. Dès lors, le choix de s'en tenir au "vivre" va de pair, ou se trouve immédiatement corrélé, avec celui de délaisser le dire : le phénoménologue revient donc aux vécus dans la pleine conscience que cette flexion en retour implique l'abandon du langage. Car c'est l'évidence de cette antinomie existante au principe du rapport entre les phénomènes et la parole que le projet phénoménologique se doit de prendre à cœur : de ce fait, le défi en la matière consiste à examiner les choses en se passant du langage. Et même : tout le projet est de tourner le dos au discours afin de s'offrir la possibilité de s'inquiéter des vécus - c'est du moins ce que l'on peut conclure à partir de cette affirmation de Heidegger : "La recherche philosophique devra ici renoncer à une "philosophie du langage" pour s'enquérir des "choses mêmes" $\gg^{25}$. C'est que la relation d'opposition instaurée entre les phénomènes et la parole se fait sur le fond d'une réciprocité : aussi, si l'attention aux choses suppose une inattention aux mots, réciproquement, la considération du langage s'accompagne d'un oubli de l'apparaître. En l'occurrence, $c^{\prime}$ est ce que prouve toute position qui s'attache à privilégier le discours : ce privilège, assurément, ne peut s'établir qu'au détriment des apparitions. De ce fait, puisque la parole est l'adversaire ou l'opposant de l'expérience, qui la renie de s'imposer, alors le désir phénoménologique d'une remontée aux vécus prend la forme $d$ 'une négation de l'expression : par conséquent, le phénoménologue ressaisit le "vivre" de telle sorte qu'il s'adonne, par là même, à une dénonciation du dire. En somme, "Le langage permet [...] de renvoyer à ce qui n'est pas, ne peut être donné, et c'est généralement contre lui que l'on énonce la nécessité du retour au donné ${ }^{26}$.

Plus précisément, la tâche qu'endosse le phénoménologue admet une certaine particularité : il s'agit, en toute rigueur, de nier un certain type d'expression. Celle qu'élabore la philosophie traditionnelle et qui a pour marque, justement, d'être inattentive à l'expérience : ainsi, la phénoménologie se veut être une négation du langage compris comme langage de la philosophie au sens institutionnel - en tant qu'il possède, par excellence, ce défaut consistant à s'imposer sous la forme d'un détournement des vécus : en tant qu'il s'en tient aux mots de ne pas s'en tenir aux choses et, radicalement, de s'en détourner. Mais qu'entendre par ces affirmations ? Pour saisir ce qui cherche à voir le jour, il convient d'abord de considérer - au moins brièvement - ce que recouvre la parole philosophique ici mise en question. Il n'est que de s'en remettre à l'impression première et

\footnotetext{
24. J. Patočka, Qu'est-ce que la phénoménologie?, Grenoble, Jérôme Millon, coll. «Krisis », 1988, trad. E. Abrams, p. 98.

25. M. Heidegger, Etre et temps, op. cit., p. 166.

26. J. Benoist, L'idée de phénoménologie, op. cit., p. 58.
} 
frappante qui saisit tout lecteur en philosophie : tout se passe alors de telle sorte que l'on se sent confronté à une évidente abstraction terminologique et à une discursivité qui semble bel et bien se construire $s u b$ specie aeternitatis. C'est dire que le philosophe élabore un univers langagier dont la caractéristique demeure dans la mise à l'écart de toute référence à l'expérience concrète : de fait, "Les textes philosophiques sont parfois si denses et si abstraits qu'il semble impossible de les rapporter à une expérience vécue ${ }^{27}$. En bref, l'expression dont il s'agit ne se fonde pas sur un renvoi à la sphère du "vivre" : à l'inverse, elle se construit par cette absence de renvoi. Cela signifie que l'essentiel en le langage philosophique réside en le langage même, ou plutôt : c'est dans les mots que tout se fixe - dans les mots mis à part leur référence. La parole prend donc le pas sur les phénomènes : autrement dit, la philosophie donne l'avantage à l'expression, en effaçant la possibilité de se soucier des vécus - "En philosophie $[\ldots]$ on ne part guère que des mots » de telle sorte que «le travail philosophique en tant que tel, pas plus qu'il ne consiste à fabriquer les structures par quoi la science représente un monde à manipuler, ne vise [...] un vécu $»^{28}$. En l'occurrence, c'est cette façon de procéder que le phénoménologue a pour ambition de remettre en cause : il développe un soupçon ou un doute à l'égard d'un tel usage langagier. Car cet emploi a pour principe l'idée que la parole n'admet aucune ouverture possible, et est toujours déjà - au contraire - fermée sur son propre espace. C'est ce qu'atteste, effectivement, l'affirmation selon laquelle les mots ne se refèrent pas à l'expérience: cela veut dire, en définitive, qu'ils ne se rapportent qu'à eux-mêmes - dans une complète fermeture à l'égard de tout ce qui leur est extérieur. Les mots révèlent par conséquent l'évidence de leur propre clôture, dès lors qu'ils s'offrent comme dépourvus de toute ouverture sur les choses elles-mêmes - comme privés de la possibilité joyeuse d'y prendre leur source ou d'y trouver leur horizon. Tel est le tort du langage philosophique: se fonder sur le postulat que l'expression ne se déploie qu'au sein de son propre espace, clos par nature. Or, le phénoménologue formule à ce niveau une proposition tout à fait opposée : selon lui, le discours a pour point de départ, de même que pour point de vue, les choses elles-mêmes. Il ne s'origine pas en son propre intérieur, et $n^{\prime} y$ retourne pas non plus - par le fait d'un fléchissement vers soi : à l'inverse, l'impulsion du langage provient des vécus, vers lesquels il est également constamment question de se diriger. Aussi, la textualité phénoménologique ne cesse de souligner son absolue déhiscence : d'un bout à l'autre, son sens propre consiste en une complète ouverture à l'expérience. C'est toute la signification

\footnotetext{
27. F. Cossutta, Éléments pour la lecture des textes philosophiques, Paris, Bordas, 1989, p. 61.

28. Y. Belaval, Les philosophes et leur langage, Paris, Gallimard, 1952, p. 148 et G.-G. Granger, Essai d'une philosophie du style, Paris, Odile Jacob, 1988, p. 302.
} 
de cette affirmation husserlienne selon laquelle "nous ne voulons absolument pas nous contenter de "simples mots", [...]. Nous voulons retourner aux "choses elles-mêmes" $»^{29}$.

Ainsi la phénoménologie reproche-t-elle au langage de la philosophie traditionnelle sa tendance à focaliser sur l'expression par une omission de l'expérience : outre cela, le phénoménologue témoigne d'une réticence à l'égard du langage en question par ceci qu'il se plaît à recouvrir les phenomènes au moyen de la parole. C'est ce qui illustre à nouveau cette occultation des choses par les mots dont fait preuve le philosophe: car cette dissimulation du "vivre" sous la sphère langagière représente une possibilité parmi d'autres d'éclipser le champ phénoménal. En ce sens, le phénoménologue prolonge à ce sujet sa critique de la parole philosophique : il s'oppose radicalement à cette façon de masquer les vécus derrière une couche de langage. Tout son projet est, justement, de dévoiler ce qui tend ici à être voilé - et manifestement obscurci : il veut s'offrir l'apparition de l'apparaître et ce, en remettant en cause toute propension dissimulatrice. De ce fait, la phénoménologie s'attache à ressaisir les choses par-delà l'expression qui les cache et les couvre: elle retourne aux phénomènes de telle sorte qu'elle plonge en leur cœur, tels qu'ils sont donnés dans toute leur fraîcheur et virginité - tels qu'ils se livrent avant même que le langage ne les ai dérobés à la vue. Aussi, pour citer E. Fink, la tâche du phénoménologue consiste alors à "effacer l'empreinte [...] inaperçue, que toute chose et tout ce qui est en général a reçu de la sphère de sens linguistique depuis des temps immémoriaux $»^{30}$. Cet engagement au sein des choses elles-mêmes - offertes dans leur pureté éclatante et en amont de toute opacité langagière - fonde donc une nouvelle fois la critique phénoménologique du discours de la philosophie traditionnelle.

Mais ce n'est pas tout: le reproche formulé par le phénoménologue porte également sur l'usage de l'argumentation en philosophie. $C^{\prime}$ est qu'il s'agit d'un procédé discursif au service, précisément, de ce désir d'occulter l'expérience : car il ne va pas sans l'idée d'une généralisation, qui est par définition une négation de toute singularite. Or, on le remarque au cours de la variation eidétique, la phénoménologie est attentive aux vécus de telle sorte qu'elle veut en ressaisir l'essence, laquelle s'accorde avec le singulier dans un refus corrélatif du général. Le retour aux phénomenes exclut donc tout processus qui consiste à généraliser, et a fortiori ceux dont la nature est langagière : il les chasse du fait qu'ils représentent, strictement, sa propre annulation. Parce que généraliser, en effet, c'est se détourner des phéno-

\footnotetext{
29. E. Husserl, Recherches logiques, tome 2 : «Recherches pour la phénoménologie et la théorie de la connaissance », première partie (Recherches I et II), op. cit., p. 5-6.

${ }^{30}$. E. Fink, De la phénoménologie, Paris, Les Éditions de Minuit, coll. "Arguments », 1990, trad.

D. Franck, p. 212.
} 
mènes, loin d'y revenir : plus précisément, c'est détourner ses yeux de la singularité caractéristique du champ phénoménal - et en nier, par là, la quintessence même. Ainsi, la tâche phénoménologique d'une flexion vers l'apparaître s'accompagne forcément d'une critique de la philosophie traditionnelle en tant qu'elle emploie l'expression argumentative, laquelle voile l'expérience singulière en $s^{\prime}$ adonnant à la généralisation. Tel est, en somme, le mot de Husserl : la phénoménologie prend corps dès que, " au lieu d'argumenter generaliter, on en vient aux choses elles-mêmes $»^{31}$.

En l'occurrence, cette critique du langage philosophique, ici restituée de manière assez schématique, est tout à fait révélatrice : elle conduit, effectivement, à modérer l'ensemble du propos dernièrement soutenu. Car elle signifie bel et bien que la phénoménologie ne fait pas le choix des phénomènes contre celui des mots : son immersion dans l'apparaître ne constitue pas, en toute rigueur, un désengagement radical à l'égard du langage. Certes, le "vivre" n'est pas absolument dicible - il comporte, on l'a noté, une part d'ineffable irréductible : mais il n'est pas, pour autant, totalement indicible - il détient, on l'a vu aussi, une sphere vouée à l'expression d'elle-même. Dès lors, s'offrir à l'exploration des choses mêmes ne suppose pas forcément plonger au cour d'un silence indépassable : seul un certain pan demeure muet et s'oppose à tout basculement dans la parole. $C^{\prime}$ est justement cette nuance qui se dessine ici, sous la forme d'un passage du mutisme total à l'ineffable partiel ou du tout à la partie, que revèle la critique du langage de la philosophie traditionnelle: parce que cette critique repose sur ce même glissement. C'est qu'elle rend manifeste le fait que le phénoménologue ne rejette pas tout langage, mais seulement un certain type de langage : elle dévoile qu'il ne choisit pas les choses de ne pas choisir le langage, mais plutôt - et plus subtilement - qu'il choisit les choses de ne pas choisir un certain langage. Celui, proprement, qui s'impose sous la forme d'une occultation de ces mêmes choses. L'exclusion phénoménologique du langage que l'on s'est attaché à souligner ne débouche donc pas sur " une complaisance dans l'ineffable $»^{32}$ : elle aboutit seulement au refus d'un langage particulier et déterminé. Car, après tout, la textualité demeure nécessaire, et ne cesse pas de s'imposer dans sa nécessité : la phénoménologie étant une discipline langagière par définition, elle ne peut s'appliquer à abolir toute expression - elle exclut simplement cette sphère expressive dont l'essence consiste en une contradiction de son propre projet d'un retour aux choses

\footnotetext{
31. E. Husserl, Recherches logiques, tome premier : «Prolégomènes à la logique pure », Paris, Puf, coll. «Épiméthée», 1969, trad. H. Élie, A. L. Kelkel et R. Schérer, cité d'après la pagination allemande, p. 155.

32. N. Depraz, Écrire en phénoménologue, op. cit., p. 103.
} 
mêmes. Ainsi met-elle uniquement à l'écart la parole employée par le philosophe traditionnel.

C'est dire que le champ phénoménologique, loin d'être d'un bout à l'autre promis au silence, conserve une vocation à la dicibilité : être phénoménologue, c'est savoir faire sa part au langage. Or, si un certain type de discours est ici écarté, lequel est retenu?

\section{Un langage respectueux des phénomènes}

$C^{\prime}$ est à ces questions qu'il faut désormais tenter de répondre, en $\mathrm{s}^{\prime} \mathrm{y}$ engageant avec le projet de découvrir en elles ce qui résiste encore au dévoilement.

Pour commencer, il n'est que de plonger dans l'idée selon laquelle la phénoménologie admet un certain langage : ainsi, soulignons encore une fois qu'elle ne s'oriente pas vers les choses par un mouvement de suppression de l'expression - sans quoi « il y aurait deux mondes : celui de ce qui est dit et celui du donné. Or cela va contre l'évidence qui constitue le fond même de notre vie : nous vivons dans un seul et même monde ${ }^{33}$. Il faut donc soutenir, à l'inverse, que le phénoménologue fléchit vers l'expérience tout en conservant la discursivité, mieux : tout en retenant un langage particulier, qu'il retient de faire sien et d'élire au titre de langage phénoménologique. En l'occurrence, ce discours est adopté, si tant est qu'il endosse une particularité : celle de s'accorder avec le fléchissement phénoménologique vers le champ phénoménal, plutôt que de le nier. Autrement dit, le phénoménologue est un être de langage à condition que les mots dont il use renforcent sa vocation à l'apparaître, au lieu de la contredire. Aussi, il se dirige vers l'expérience tout en s'exprimant, mais à ceci près que son expression doit être la cristallisation de cette orientation vers les vécus : en ce sens, il est possible de dire en phénoménologie si, dans le même temps, on s'immerge dans le "vivre" ; si, simultanément, on fait de la discursivité l'incarnation du retour aux choses. Par conséquent, il n'y a plus alternative en phénoménologie entre le fait de dire et celui de vivre dès lors que la diction cesse de contredire la vocation à l'apparaître, et se propose de la renforcer au contraire : dès lors, en somme, que l'expression n'est pas contre le retour aux phénomènes, mais à la mesure de ce retour - à la mesure, tout simplement, des phénomènes vers lesquels il s'agit de se retourner. Mais quelles caractéristiques endosse cette expression à l'échelle des phénomènes? Quelle est donc cette discursivité que le phénoménologue retient comme sienne, qu'il élit et dont il use, du fait qu'elle est à la mesure de l'expérience - et en mesure, par conséquent, d'exprimer cette même expérience sans la contredire? En un mot : quelle est sa teneur?

33. J. Benoist, L'idée de phénoménologie, op. cit., p. 58-59. 
Husserl, on va le voir, peut nous apporter ici quelques éclairages. Partons du postulat, avancé par Natalie Depraz, selon lequel «l'ecriture qui se donne pour tâche d'épouser [la réalité des phénomènes] au plus près se doit de la respecter $»^{34}$ : ce postulat permet d'avancer dans l'analyse, parce qu'il laisse sensiblement supposer que le discours qui est à la mesure de l'apparaître a pour teneur propre celle d'être respectueux de cet apparaître. Il laisse à penser, en d'autres termes, que le phénoménologue fait droit à une expression dont la spécificité est de nourrir des égards à l'égard de l'expérience ; dont la quintessence, plutôt, est de rendre respect aux choses qu'elle exprime. Ceci étant dit, il n'empêche que le mystère demeure quant à cette quintessence : car que signifie précisément la notion de "respect" ici en question? Elle signifie que le phénoménologue ne veut pas ajuster les phénomènes à son langage mais, au contraire, ajuster son langage aux phénomènes. Expliquons point par point. Parler phénoménologiquement, c'est parler de l'expérience d'une parole qui contient ce qu'elle thématise : seulement, cette concentration du vécu au sein des multiples branches langagières n'est pas le fait d'une agressivité exercée à l'endroit du phénoménal. En effet, le langage ne s'essaie pas alors à une tentative de reconduction à soi de l'expérience : il ne souhaite à aucun moment lui imprimer une pression ou la contraindre à entrer dans son champ d'appartenance. De cette façon, la diction phénoménologique des choses mêmes ne prend pas la forme $d^{\prime}$ une violation de celles-ci : puisqu'elle n'est pas un mouvement consistant à les ramener à soi par le fait d'un désir de l'enserrer dans ses serres ; puisqu'elle ressaisit les vécus, donc, d'un ressaisissement qui ne leur fait pas violence. Ainsi y a-t-il respect puisqu'il n'y a pas tentative d'ajustement des choses aux mots : de fait, le langage dont use le phénoménologue captive ou absorbe les vécus certes, mais en les respectant toujours - c'est-à-dire en ne s'exerçant jamais à les trahir ou à les déformer par une réduction de ces vécus, justement irréductibles, à sa propre nature. Davantage, il faut bien reconnaître à ce niveau que l'expression phénoménologique témoigne d'un souci de l'expérience qu'elle exprime : elle en est soucieuse, en effet, parce qu'elle traite des phénomènes dans le refus même de les altérer ou de les déformer par ce traitement ; parce qu'elle n'en propose pas une déformation ou à une altération, mais s'évertue plutôt à les accueillir et à se tenir à leur écoute. C'est dire que l'expression phénoménologique fait preuve de soin et de délicatesse vis-à-vis de ce qu'elle s'attache à restituer et à traduire en mots : la prudence et la vigilance la dirigent attendu qu'elle s'efforce, autant que possible, de ne pas heurter le donné; attendu qu'elle se prête à dire ce donné avec la garantie qu'elle ne le mettra pas à son service. C'est dire, surtout, que le

34. N. Depraz, Écrire en phénoménologue, op. cit., p. 167-168. 
phénoménologue fait justement tout pour mettre son discours au service des phénomènes: ainsi peut-on en déduire qu'au lieu d'ajuster le "vivre" au "dire", il exprime l'expérience en ajustant son expression à cette expérience même. Car il fait en sorte qu'elle soit fidèle au fond tout autant qu'à la forme de l'apparaître : il fait en sorte qu'elle soit adaptée aux vecus - qu'elle s'impose sous la forme d'un "dire" approprié au "vivre". Tel est le sens rigoureux du respect que l'expression phénoménologique entretient à l'égard de l'expérience : on le voit, il s'agit d'un respect des choses elles-mêmes qui consiste en le désir de restituer avec justesse ces choses ; en l'ambition, même, d'instituer une parole qui ne s'en écarte pas - qui refuse de se les soumettre pour plutôt $\mathrm{s}^{\prime} \mathrm{y}$ soumettre, en s'accordant avec leurs rythmes, avec leurs qualités : en un mot, avec leur texture. Tout fait donc fond ici sur l'adoption d'une attitude manifestement éthique : Natalie Depraz le soutient d'ailleurs, puisqu'elle avance à propos de Husserl que "le phénoménologue tient un propos prescriptif, qui répond à une éthique du langage "propre" ou "adéquat" ${ }^{35}$. Au reste, et ceci suffira à justifier l'ensemble de notre analyse, cette exigence d'adéquation ou de fidélité vis-à-vis des apparitions est, on l'a déjà noté, soutenue par Husserl lui-même : selon lui, le dire ne peut ramener à soi le donné jusqu'à le faire jaillir au milieu des mots, qu'à la condition de s'accorder à l'ensemble de ses traits distinctifs et de s'y conformer. En somme, «quand l'expression [...] est rapportée à l'objet donné, l'objet se constitue alors comme "donné" dans certains actes, et, de fait, est donné en eux - si toutefois l'expression s'ajuste réellement à ce qui est donné $\gg$ 36.

Ainsi est-on davantage renseigné quant à la teneur du langage dont use le phénoménologue: il s'agit d'un langage respectueux, d'un respect qui consiste en ce que les mots s'annexent aux choses plutôt que de se les annexer. Mais allons plus loin dans cette perspective : l'idée est ici que la discursivité ne s'impose pas face à l'ensemble de ce qui se phénoménalise, et qu'elle refuse de violenter les phénomènes en prenant pour visage celui d'une entité envahissante. Ajoutons désormais que cette même discursivité, loin de seulement opter pour la non-violence, opte aussi pour l'effacement et le retrait: elle se replie en présence du phénoménal, et ce, en cherchant à s'éclipser. Par le fait, le retirement du langage laisse alors aux choses la possibilité inverse de s'avancer: celles-ci découvrent qu'elles peuvent s'affirmer là où, précisément, l'expression recule. En l'occurrence, la forme expressive retenue pour dire l'apparaître, loin d'être mise en place par la parole, l'est en fait par l'experience : c'est à elle que revient, en définitive, le pouvoir de décider ce qui est en

\footnotetext{
35. N. Depraz, Lire Husserl en phénoménologue, op. cit., p. 177.

36. E. Husserl, Recherches logiques, tome $2:$ «Recherches pour la phénoménologie et la théorie de la connaissance », première partie (Recherches I et II), op. cit., p. 50-51.
} 
mesure ou non de la traduire. Cela implique qu'elle détient une puissance de direction vis-à-vis des mots : elle bénéficie d'une prérogative à leur égard, puisqu'elle les conduit - dans le refus de se laisser mener par eux. Par là même, on voit que la phénoménologie donne un tour radical à ce rapport ici analysé entre l'expérience et l'expression : parce qu'il semble bien qu'à son sens les vécus ne sont pas réduits à l'expression au sens fort où c'est plutôt cette dernière qui se voit reconduite à l'espace du "vivre". La diction du donné, effectivement, ne manie pas ce donné en se l'asservant : au contraire, la donation est dicible d'une dicibilité qu'elle modèle et façonne à sa propre dimension. Aussi, il faut en conclure que la préséance ne revient pas à la sphère langagière de revenir, en définitive, à celle de l'expérience - pour prendre appui sur Husserl, citons simplement : la phénoménologie ne privilégie pas les mots aux dépens des choses mais, a contrario, "laiss[e] [...] le dernier mot aux choses ellesmêmes $»^{37}$.

Qu'en conclure? Que le langage phénoménologique est respectueux des choses de tomber dans un irrespect de lui-même. Sensiblement, en effet, il apparaît que le glissement se fait à ce niveau vers le risque inverse à celui relevé ci-dessus : car si la phénoménologie évite le travers consistant à soumettre l'expérience à l'expression, il n'est pas certain qu'elle ne tombe pas dans le défaut opposé d'une soumission de l'expression à l'expérience. C'est en tout cas ce qui se dessine à l'arrière-fond de toute notre analyse : la parole y paraît bel et bien dominée par les phénomènes. Puisqu'elle est présentée comme ce qui est tenu de répondre au commandement phénoménal, de sorte qu'elle ne s'oriente pas d'elle-même, mais en réponse à ce que lui dicte le champ de l'apparaître ; puisqu'il est exige, en outre, que les mots s'inscrivent dans les limites de l'espace expérientiel, de sorte qu'ils perdent une part de leur indépendance - en tant qu'ils se trouvent alors assujettis et soumis aux règles qui ne sont pas les leurs propres, mais celles fixées par l'expérience. Puisque la possibilité est, enfin, que violence soit faite au langage: au sens où celui-ci doit, littéralement, "se faire-violence" pour être à la mesure des règles en question. Laisser le dernier mot aux choses, ainsi que Husserl entreprend de le faire, c'est donc sous-entendre que la discursivité se livre a la déformation et à la défiguration d'elle-même.

Pour autant, cette conclusion radicale ne restitue pas justement ce que la phénoménologie donne à voir : de fait, elle ne cultive pas un tel assujettissement du "dire" au "vivre". C'est ce qu'il faut tenter de comprendre avec exactitude, en ressaisissant précisément ce qu'il se produit dans ce rapport phénoménologique des mots aux choses.

37. E. Husserl, Recherches logiques, tome premier : «Prolégomènes à la logique pure », op. cit., Préface de la deuxième édition, p. XIII. 
D'abord, il est certain qu'il n'y a pas de soumission de l'apparaître à la discursivité, mais il est certain également qu'il en va de même dans le sens inverse - aussi, il n'y a en fait pas d'asservissement de la parole aux phénomènes. Toujours est-il que la textualité phénoménologique ne cesse pas de s'accorder avec l'ambition consistant à donner le dernier mot aux choses : simplement, il n'est pas question de voir dans ce modelage du texte à la mesure des vécus une forme d'assujettissement du langage à l'expérience. Car la phénoménologie, pleinement comprise, ne porte pas la sphère langagière à un rang subalterne - par le fait d'une diminution de son importance et, en somme, d'un amoindrissement ou d'une déconsidération de son être. Bien au contraire, le plan phénoménologique ne se propose jamais que de surélever le langage en le portant a la hauteur d'une exigence rigoureuse : tout se passe donc ici à la lumière de l'ambition voulant exhausser les mots, et non pas les repousser à un rang secondaire. Parce qu'en définitive confier au langage la tâche de dire les phénomènes, c'est laisser reposer en lui beaucoup d'espoir - et lui accorder en tout cas beaucoup de confiance. C'est bel et bien le conduire à la possibilité d'un "plus-être", et non le rabattre vers un "moindre-être" : en bref, c'est formuler le souhait de l'élever en s'opposant à toute volonté de le rabaisser. Ainsi la phénoménologie, selon J.-Y. Pouilloux et M. Dufrenne, met-elle toute sa « confiance [...] dans une écriture susceptible de ramener au jour ce qui constitue l'épaisseur méconnue de l'expérience $»^{38}$ - disposée qu'elle est à voir en son écriture sa «vertu d'expressivité » "son pouvoir d'exprimer la force de l'apparaître $»^{39}$ et non pas ses défauts ni son impuissance.

En bref, le respect des phénomènes dont fait preuve le langage phénoménologique ne conduit pas à un irrespect de lui-même en tant que langage. Mais, ceci étant clarifié, un problème se pose encore : car comment les mots peuvent-ils respecter les choses elles-mêmes jusqu'au bout? Comment le peuvent-ils, en effet, puisque ces choses ont une part d'ineffable; puisque les dire c'est en ce sens déjà les contredire, du moins contredire leur versant silencieux ? Si le langage ne tombe pas dans un irrespect de lui-même, il semble en revanche qu'il verse dans une difficulté de taille : celle que représente la forme qu'il doit adopter pour respecter les phénomènes, compte tenu de leur indicibilité. Il est donc question, à ce stade, de préciser avec davantage d'acuité ce en quoi consiste la teneur recherchée de l'expression phénoménologique : car, on l'a vu, sa caractéristique est d'être une expression qui respecte les choses, mais encore faut-il

\footnotetext{
38. «Je ne sais ce que je vois qu'en écrivant ", J.-Y. Pouilloux, in Merleau-Ponty et le littéraire, textes réunis et présentés par A. Simon et N. Castin, Paris, Presses de l'École normale supérieure, 1997, p. 100.

39. M. Dufrenne, Esthétique et philosophie, tome 1, Paris, Éditions Klincksieck, coll. d'esthétique, 1967, p. 112.
} 
déterminer ce qui fait sa marque dès lors que l'on prend en compte l'indicibilité de ces choses qu'elle respecte. Disons-le simplement : sa spécificité est alors en ceci qu'elle va prendre la forme d' "une discursivité à la limite". Qu'est-ce à dire ? Expliquons.

Il a été souligné, mais revenons y rapidement, que tout un pan de l'expérience releve de l'ineffable : selon Husserl, en cette affirmation déjà citée, le phénoménal n'est jamais que « l'expérience pure et, pour ainsi dire, muette encore ${ }^{40}$. Le vécu est donc indicible par tout un côté, même s'il ne l'est pas totalement: par ce côté rendu manifeste au travers de l'épaisseur des choses elles-mêmes. Le champ de l'intraduisible, en effet, n'est jamais que cet ensemble d'éléments de l'expérience auquel la clarté et la translucidité font défaut: si le "vivre" n'est pas totalement saisissable par le langage, c'est donc en cela que subsiste toujours en lui des singularités obscures et denses - dont la caractéristique est, justement, de ne pas être traduisible en mots. De fait, seuls certains aspects du vécu sont mis en lumière par la parole, ce qui suppose qu'un certain nombre d'autres n'en sont pas dévoilés : ceux qui sont précisément, de par leur dimension flottante et enchevêtrée, voués à rester dans l'ombre. De fait encore, même les versants traduisibles de l'expérience - offerts sous la forme d'un condensé de sensations et de pensées précises, claires, et même définies - surgissent d'un fond confus dans lequel tout est mélangé, d'un mélange indicible. C'est dire que les phénomènes participent de l'ineffable en raison de leur tendance à faire preuve d'indécision : leur silence a pour cause, en somme, leur tournure vague et oblique. De la sorte, c'est parce que l'expérience présente multiples plis et plissements troubles, c'est parce qu'elle est composée et non simple, que le langage n'est pas en mesure de l'englober totalement: en ce sens, le mutisme vaut pour le vécu brut ; pour ce vécu dépourvu de netteté et qui échappe par là même à la possibilité d'être limité par des contours possibles - en l'occurrence : par les frontières étroites du langage. C'est précisément là que réside toute la difficulté de la tâche du phénoménologue puisque, s'il veut dire les phénomènes, il doit forcément mettre en place une diction qui s'ajuste à leur indicibilité : une diction dont « le sens », par conséquent, "prend la densité, mais aussi l'opacité, des choses $» 14$. Cela signifie que le phénoménologue doit créer une textualité qui, paradoxalement à elle-même, côtoie l'indicible : car, pour s'appuyer sur Husserl, «c'est l'expérience pure et, pour ainsi dire, muette encore, qu'il s'agit d'amener à l'expression pure de son propre sens $»^{42}$, sachant que l'articulation produite s'impose alors en partie comme une formulation de l'inarticulable - ou, en tout cas, de l'inarticulé. Dire les phénomènes, c'est donc dire ce qui

40. E. Husserl, Méditations cartésiennes, op. cit., p. 73.

41. M. Dufrenne, Esthétique et philosophie, tome 1, op. cit., p. 152.

42. E. Husserl, Méditations cartésiennes, op. cit., p. 73-74. 
est exprimable tout en ne l'étant pas - c'est accorder la diction avec une part d'indicible: c'est en ce sens rigoureux que le langage phénoménologique est bel et bien "un discours à la limite" puisque confronté ou conduit, on le voit, à sa propre limite.

Mais est-ce que cette limite est un défaut pour autant? En d'autres termes, cristallise-t-elle une défaillance du langage? Il semblerait, à première vue, que la parole soit ici concrètement mise face à ses propres faiblesses : 1 'idee que le langage traduise en mots l'expérience tout entière, y compris son versant ineffable, paraît relever seulement de l'idéal. Tout porte à croire, en effet, que la diction des choses mêmes, comprise comme diction d'un exprimable mêlé d'inexprimable, est à renvoyer du côté du rêve ou de l'utopie : par conséquent, le phénoménologue serait tenu à ce niveau d'admettre qu'il est « "en manque d'expression" $\gg^{43}$. Néanmoins, c'est là se fermer un peu rapidement le champ des possibles: parce qu'en définitive il n'est pas radicalement impossible pour la phénoménologie d'instituer un langage à la mesure de cette dialectique expérientielle entre le "dire" et le "taire". Il faut pouvoir envisager, à ce niveau, la création d'une discursivité capable de contenir en elle les vécus, dans tout ce qu'ils ont de manifestement dicible et silencieux. Ainsi ne doit-on pas exclure l'idée que la sphère phénoménologique puisse posséder cette aptitude à mettre en mots le "vivre" - c'est-à-dire à porter à l'expression cette alliance de dicibilité et d'incessant passage sous silence : en bref, il convient d'accorder sa confiance à la courageuse présomption phénoménologique d'un langage du phénoménal, exprimant tout à la fois ce qui est voué à être dit et à être tu. La possibilité est donc, et l'on peut se permettre d'y croire, qu'il existe une " "langue phénoménologique pure" » dont tout le sens est d'être un "dispositif approprié à l'enregistrement [...] des matériaux d'expérience qui [...] constituent de véritables réalités ineffables $»^{44}$.

Dès lors, si un langage à la limite est possible, en quoi consistera-til précisément? Il n'est que de se représenter un langage en adéquation avec la richesse expérientielle, dont la luxuriance repose dans ce subtil mélange d'articulation et d'inarticulation : le tout est d'éviter ce travers du discours qui consiste à s'imposer dans son autorité et sa force - force d'identité et de clarté. Parce que c'est justement ce type de discursivité qui conduit à l'objection soulignée plus haut, celle d'une impuissance langagière à dire les phénomènes : en ce cas, oui, il est vrai que la parole ne peut pas exprimer l'expérience comprise comme dicible-indicible, en cela qu'elle la heurte en l'astreignant à sa positivité et à son exactitude. Elle perd, en somme, cette part fuyante et relativement insaisissable du phénoménal : de ce fait, elle rencontre

43. P. Loraux cité in A. Soulez, Comment écrivent les philosophes, op. cit., p. 102.

44. Ibid., p. 149. 
sa propre limite. Car une partie du champ expérientiel lui échappe alors - ou plutôt : échappe à cette volonté langagière qui veut muer les choses elles-mêmes en choses thématisees et réduites à un ensemble de significations strictement instituées. Le discours bute contre sa faiblesse, en découvrant qu'il ne parvient pas à saisir l'expérience : mais c'est qu'il doit précisément abandonner toute tentative de la capturer - parce que c'est ainsi qu'il la perd. Si la discursivité souhaite ne pas défaillir à ce niveau et appréhender l'expérience jusqu'au bout d'elle-même, alors elle doit renoncer à toute idée de possession et se livrer au contraire au désir d'approcher, ainsi que le propose M. Richir, la " "chose" (Sache), la véritable Sachlage phénoménologique, à laquelle le dire $[\ldots]$ du langage pris dans sa dimension phénoménologique peut seulement toucher sans l'enfermer, sous peine de la faire disparaître dans les cadres expressifs de la parole articulée $\gg^{45}$. Telle est donc la forme que prend la parole phénoménologique, dès lors qu'elle a pour vertu d'être véritablement en adéquation avec la réalité articulable-inarticulable des phénomènes : elle s'entend comme une discursivité à sa limite, qui découvre non pas ses failles mais le champ phénoménal situé aux frontières du discours - sur la ligne de crête entre le langage et le non-langage. La textualité phénoménologique parvient, de ce fait, à exprimer les choses elles-mêmes et à les embrasser - jusque dans leur intimité fuyante. Elle aboutit à l'horizon de son dessein, qui est de dire les vécus en les respectant, même là où ils basculent dans l'ineffable. Cela signifie que le phénoménologue, si tant est qu'il veut réaliser l'objectif qu'il s'est fixé, n'est jamais soumis qu'à une seule condition : celle de s'exprimer, pour reprendre une nouvelle fois les mots de M. Richir, dans la pleine conscience qu'il cherche alors "à dire "quelque chose" un tant soit peu complexe, nuancé ou subtil, et qui échappe, nous le sentons, à la clarté de l'expression ${ }^{46}$. Il ne doit jamais perdre de vue le fait que la matière à traiter s'inscrit aux bordures du dicible, frôlant l'espace du silence : il est tenu de s'en tenir à l'évidence de sa confrontation à un champ presque ineffable - en équilibre entre la dicibilité et le mutisme. Ainsi le phénoménologue a pour exigence, unique entre toutes, de ne jamais oublier ni omettre la nature glissante de l'expérience : en somme, il faut qu'il garde en mémoire, comme le souvenir le plus précieux et le plus essentiel en la circonstance, l'essence du phénoménal - laquelle est prête à jaillir dans ses mains, si tant est qu'il accepte la possibilité, toujours menaçante et merveilleuse, qu'elle file entre ses doigts.

45 « Phénoménologie, métaphysique et poïétique », M. Richir, op. cit., p. 96.
46 Ibid., p. 91. 